\title{
NOVEL DRUG DELIVERY SYSTEMS: AN OPPORTUNITY FOR PHARMACEUTICAL SCIENTISTS IN INDIA
}

\author{
Dear Reader,
}

Innumerable drug delivery systems are available for the introduction of the drugs into the body to treat various diseases, to enhance the therapeutic efficacy and to improve patient compliance. These systems include oral controlled systems, parenteral systems, nasal and pulmonary systems, transdermal systems, nanoparticles, dendrimers, lipid based systems (liposomes, nanostructured lipid carriers, etc), self-regulated systems and

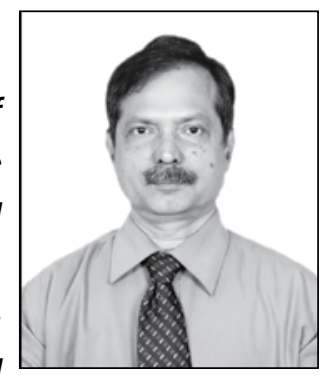
targeted drug delivery systems.

The rapid advancement of size evaluation technologies has led to the development of nanoparticulate systems which are used in diagnostics, treatment, drug delivery, nutraceuticals, cosmeceuticals and in the production of bio-compatible materials with improved properties. The reason for the appeal of nano-particulates formedical purposes is because of their exceptional features such as large surface area, ability to carry other molecules and absorptive nature. Besides having large functional surfaces, nanoparticles can also be used to deliver the drugs to target sites and thus reduce toxicity to the non-target organs. Nano-particulates may be i) inorganic nanoparticles (carbon nanotubes, ceramic nano-particulates, calcium phosphate nano-particulates, gold and silver nanoparticles, magnetic nanoparticles, quantum dots, etc and/or ii) organic nanoparticles (polymeric micelles, solid lipid nanoparticles, polymeric nanoparticles, nano-emulsions, liposomes, dendrimers, etc.). Liposomes, Ethosomes, Transfersomes, Archaeosomes, Pharmacosomes, Solid lipid nanoparticles, Nanostructured lipid carriers, Lipid nano-capsules and Lipospheres etc are the examples of lipidic nanoparticles. Intestinal protective drug absorption system(IPDAS), SpheroidalOral Drug Absorption System (SODAS), Programmable Oral Drug Absorption System (PRODAS), Diffucaps, Eurand MINITABS, Macrocap ${ }^{\circledR}$, Orbexa ${ }^{\circledR}$ technology and Flashtab technology are the examples oforal controlled drug delivery technologies developed commercially. Self-regulated drug delivery systems include $\mathrm{pH}$-sensitive drug delivery systems, Thermo-Responsive drug delivery systems, Competitive Binding Delivery Systems, System utilizing antibodies, System utilizing Chelation and Inflammation-induced drug delivery systems. Nowadays, novel drug delivery systems such as liposomes, cyclodextrin complexes, micro-emulsions, nano-suspensions, nano-emulsions etc. are also used in parenteral drug delivery.

Transdermal drug delivery systems include various types of patches which use active transport mechanisms such as electroporation, sonophoresis, iontophoresis and magnetophoresis. Targeted drug delivery aims to deliver the drug to the exact location where it is required. However, there are many challenges in developing novel drug delivery systems such as: lack of drug release in specific areas in a controlled manner, the construction of biocompatible and biodegradable materials, difficulties to achieve multi-function responses (active targeting, on-command delivery, intelligent devices, bio-responsive triggered systems, self-regulated systems, systems interacting with the body, smart delivery with a single system), difficulties in tissue engineering and delivery of proteins/peptides, etc. However, it is expected 
that development of novel, sophisticated, multifunctional systems which can recognize cancer cells, deliver drugs to target tissues and aid in reporting outcome of therapy would be developed in the near future. As the complexity of the drug delivery systems increases, the scale-up and commercial manufacturing processes will also require greater efforts to ensure that a product with reproducible performance characteristics is manufactured consistently. Also, more resources would have to be dedicated to obtain regulatory clearances for these complex systems. However, these efforts will result in greater benefits to both the patient and the pharmaceutical industry.

Dr Nayanabhirama Udupa

Director-Research (Health Sciences), Manipal

Email:n.udupa@manipal.edu

\section{About The Guest Editor}

Dr N. Udupa, Professor, and Director, Research (Health Sciences), Manipal University, Manipal, Karnataka, received his Ph.D. in 1987 from Banaras Hindu University, Varanasi, India. After working in the pharmaceutical industry for eight years, he moved to academics and has been working at the Manipal College of Pharmaceutical Sciences, for the last 30 years. Dr Udupa is a prolific researcher and author with more than 500 research and review articles. He has made presentations at over 400 national and international conferences and has authored about 20 books. He is on the editorial boards and advisory bodies of several reputed journals including INDIAN DRUGS. He is a life member of several professional associations and societies and has nine Indian patents to his credit. He has won many awards for his outstanding contributions in Pharmaceutical Research and Education and has served as the Convener, Scientific Services of the Indian Pharmaceutical Congress from 2010 to 2012.

它

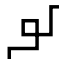

\title{
ERRATA
}

The title of the Editorial published in Indian Drugs, November 2017 issue is to be read as "Skilling Indian Scientists For The Last Five Decades" instead of "The Skilling Indian Scientists For Last Over Five Decades". The error is regretted. 